\title{
Inhibition of Trypanosoma cruzi proline racemase affects host-parasite interactions and the outcome of in vitro infection
}

\author{
Leticia Coutinho ${ }^{1,2}$, Marcelo Alves Ferreira', Alain Cosson ${ }^{3}$, Marcos Meuser Batista ${ }^{2}$, \\ Denise da Gama Jaén Batista², Paola Minoprio33, Wim M Degrave', \\ Armand Berneman ${ }^{3}$, Maria de Nazaré Correia Soeiro²/+
}

\begin{abstract}
'Laboratório de Genômica Funcional e Bioinformática ²Laboratório de Biologia Celular, Instituto Oswaldo Cruz-Fiocruz, Av. Brasil 4365 , 21045-900 Rio de Janeiro, RJ, Brasil 'Institut Pasteur, Laboratoire d'Immunobiologie des infections à Trypanosoma, Département d'Immunologie, Paris, France
\end{abstract}

Proline racemase is an important enzyme of Trypanosoma cruzi and has been shown to be an effective mitogen for B cells, thus contributing to the parasite's immune evasion and persistence in the human host. Recombinant epimastigote parasites overexpressing TcPRAC genes coding for proline racemase present an augmented ability to differentiate into metacyclic infective forms and subsequently penetrate host-cells in vitro. Here we demonstrate that both anti $\mathrm{T}$. cruzi proline racemase antibodies and the specific proline racemase inhibitor pyrrole-2carboxylic acid significantly affect parasite infection of Vero cells in vitro. This inhibitor also hampers T. cruzi intracellular differentiation.

Key words: Trypanosoma cruzi - proline racemase - host-parasite interaction - enzyme inhibition

The kinetoplastid protozoan parasite Trypanosoma cruzi is the causative agent of Chagas disease, a serious public health problem in most of the Latin American countries, and affecting about 12 million people (Dias 2007). In addition, due to migration, Chagas disease becomes a threat in several additional countries (Schmunis 2007). The parasitic illness has two main phases: the acute, which appears shortly after infection, and the chronic phase, which develops in about one-third of the infected individuals, after a silent period of years or decades. Due to this long asymptomatic state, Chagas disease is often considered a "silent killer", impairing early specific diagnosis and treatment (Tarleton 2007, Bilate et al. 2008). The main clinical manifestations of Chagas disease are cardiac and digestive alterations, and the pathogenesis has been attributed to host immune system disturbances associated with a low-grade parasite presence (Rocha et al. 2007, Marin-Neto et al. 2008). Up to now, there is no immediate prospect for the development of a vaccine and current clinical therapy based on heterocyclic nitro compounds (nifurtimox and benznidazol) is quite unsatisfactory for chronic patients, thus calling attention to the search for new therapeutic approaches (Coura et al. 2002, Dias 2006).

The life cycle of $T$. cruzi comprises distinct developmental stages such as amastigotes and epimastigotes that are multiplicative forms, found respectively in the

Financial support: CNPq, CAPES, FAPERJ, MS-DECIT, SanofiAventis/Ministère de la Recherche/Institut Pasteur (fonds dédiés 17 to Institut Pasteur and MAF)

+ Corresponding author: soeiro@ioc.fiocruz.br

Received 2 February 2009

Accepted 30 November 2009 cytoplasm of mammalian cells and within the midgut of the insect vectors and trypomastigotes, which are nondividing infective forms present in both vertebrate and invertebrate hosts (De Souza 2008).

Proline racemases are enzymes that catalyze the interconversion of L- and D-proline enantiomers and have been identified in $T$. cruzi (Reina-San-Martin et al. 2000). This enzyme was the first eukaryotic amino acid racemase described and is encoded in T. cruzi by two paralogous genes per parasite haploid genome, $T c P$ $R A C A$ and TCPRACB, that are thought to give rise, respectively, to secreted and intracellular protein isoforms (Chamond et al. 2003). The TcPRACA is an effective mitogen for host B cells thus contributing to the T. cruzi immune evasion and persistence, triggering non-specific polyclonal lymphocyte activation (Reina-San-Martin et al. 2000, Chamond et al. 2003).

In view of the fact that (i) the establishment of a $T$. cruzi infection depends on a series of consecutive biological events, involving interactions of diverse parasite molecules with host components (Yoshida 2006, Covarrubias et al. 2007, Fernandes et al. 2007), and (ii) the overexpression of TcPRAC isoforms leads to an increase of parasite differentiation into infective forms and of the efficiency of host cell penetration (Chamond et al. 2005), we decided to further address the impact of proline racemase during the intracellular cycle of $T$. cruzi besides exploring the implication of these enzymes in parasite interactions with mammalian cells in vitro.

\section{MATERIALS AND METHODS}

Mammalian cell cultures - For obtaining trypomastigotes or for the interaction assays, Vero cells (from African green monkey kidney) were seeded at a density of 1,5 x $10^{6}$ cells in $25 \mathrm{~cm}^{2}$ culture flasks or at $5 \times 10^{4}$ cells/well into 24-well culture plates containing round glass slides. The cultures were maintained in RPMI 1640 medium 
(Roswell Park Memorial Institute - Sigma Aldrich - USA) supplemented with $5 \%$ fetal bovine serum and $1 \mathrm{mM} \mathrm{L-}$ glutamine and kept at $37^{\circ} \mathrm{C}$ in an atmosphere of $5 \% \mathrm{CO}_{2}$.

Parasites - Cell culture-derived trypomastigotes from T. cruzi CL Brener (clone F11-F5) were isolated from the supernatant of Vero cells, which had been previously infected with bloodstream trypomastigotes (De Souza et al. 2003).

Infection and treatment of the Vero cell cultures Vero cell cultures were infected for $24 \mathrm{~h}$ at $37^{\circ} \mathrm{C}$ with cultured trypomastigotes at a 10:1 parasite/cell ratio in the presence or absence of increasing doses of freshly made dilutions of $10 \mu \mathrm{M}, 100 \mu \mathrm{M}$ or $1.000 \mu \mathrm{M}$ of the specific proline racemase inhibitor pyrrole-2-carboxylic acid (PYC), which was previously dissolved in dimethyl sulfoxide (DMSO). Alternatively, Vero cell lines were infected for $24 \mathrm{~h}$ at $37^{\circ} \mathrm{C}$ with trypomastigote forms in the presence or absence of 42,84 or $168 \mu \mathrm{g} / \mathrm{mL}$ of affinity purified rabbit anti-TcPRAC polyclonal IgG antibodies. The latter were produced by hyperimmunization with TCPRACA recombinant protein lacking the first 30 amino acids, corresponding to the presumed signal peptide. Mature TcPRACA and TcPRACB proteins possess $96 \%$ identity ( 7 amino acid differences). Anti-TcPRAC polyclonal antibodies recognize both isoforms of the enzyme and were used throughout the experiments. To further investigate the effect of the racemase inhibitor upon the parasite or host cells, trypomastigotes or Vero cell cultures were pre-treated separately for $2 \mathrm{~h}$ at $37^{\circ} \mathrm{C}$ with PYC $(0,10$, 100 or $1.000 \mu \mathrm{M}$ ), rinsed three times with phosphate buffered saline (PBS) and then used in a $24 \mathrm{~h}$ infection assay (10:1 parasite: host cell ratio). To verify if the proline racemase inhibitor could also exert an effect on the parasite intracellular cycle after $24 \mathrm{~h}$ of untreated parasite-host cell interaction, infected cultures were rinsed three times and incubated for up to $96 \mathrm{~h}$ with 10-1.000 $\mu \mathrm{M}$ PYC. After the different treatments, the cultures were washed with PBS, fixed with Bouin's solution and stained with Giemsa, according to AraujoJorge et al. (1989). The number of infected host cells and of parasites per infected cell was then scored in 400 host cells in three independent experiments, each run in duplicate. Results were expressed as the percentage of infected cells, the mean number of parasites per infected cell or by the endocytic index (EI) which represents the percentage of infected cells v.s. mean number of parasites per infected cell (Da Silva et al. 2008). In some assays, after the interaction for $24 \mathrm{~h}$ with PYCtreated parasites, the infected cultures were rinsed and followed up to eight days post infection. The number of parasites released into the cell culture supernatant was then counted daily. As controls, parasites were incubated only in culture medium, in medium with comparable DMSO concentrations as the samples with diluted PYC, or in the presence of non-immunized rabbit antiserum when indicated. Statistical analysis was carried out using the Student $t$-test, with the level of significance set at $\mathrm{p}<0.05$. In previous control experiments, the absence of toxicity of PYC towards Vero cells was verified incubating the inhibitor PYC at concentrations of 1-1.000 uM for 24 and $48 \mathrm{~h}$ with Vero cells and testing cell viability by Trypan Blue exclusion $(0,4 \%$ in PBS). Cells remained viable and did not incorporate the dye. No toxicity was observed. As positive controls, Vero cells were treated with $50 \mathrm{mM}$ of $\mathrm{NiCl}_{2}$ in PBS. In the latter case, dying Vero cells presented apoptotic vesicles, as examined by optical microscopy and dead Vero cells detached from the support.

Immunofluorescence - In order to analyze the expression of proline racemase during the intracellular cell cycle, Vero cells were infected with $T$. cruzi and after 2-72 $\mathrm{h}$ of infection were rinsed twice with PBS, fixed for $20 \mathrm{~min}$ at $4{ }^{\circ} \mathrm{C}$ with $4 \%$ paraformaldehyde (PFA) and washed again with PBS. The cells were permeabilized for 10 min with $0.25 \%$ Triton X-100 in PBS. Next, the samples were incubated for $30 \mathrm{~min}$ in $3 \%$ bovine serum albumin (BSA) to prevent nonspecific labeling. The cultures were then incubated for $60 \mathrm{~min}$ at $37^{\circ} \mathrm{C}$ with affinity purified rabbit IgG anti-TcPRAC diluted in PBS containing $0.25 \%$ Triton X-100 and 3\% BSA. After two washes with PBS, the samples were incubated for $30 \mathrm{~min}$ at $37^{\circ} \mathrm{C}$ with goat anti-rabbit rhodamineIgG or fluorescein isothiocyanate-IgG (FITC) (1:100, Sigma) diluted in 3\% BSA. Samples were further incubated with $10 \mu \mathrm{g} / \mathrm{mL}$ 4,6-diamidino-2-phenylindole (DAPI) to enable visualization of parasite nuclei and kinetoplasts and mounted with 2.5\% 1.4-diazabicyclo(2.2.2)-octane. As negative controls, primary antibodies were omitted or non-immune rabbit serum was used as indicated. Cultures were examined using a Zeiss Axioplan 2 microscope equipped with epifluorescence (Zeiss INC, Thornwood, NY) or with a confocal laser scanning microscope (BX51 Olympus). Epimastigote and metacyclic trypomastigote forms were treated in a similar fashion and the presence of proline racemase revealed with goat anti-rabbit tetramethyl rhodamine isothiocyanate (TRITC) or FITC and DAPI.

Ultrastructural imunocytochemistry - T. cruzi epimastigote forms, or Vero cells after $48 \mathrm{~h}$ of infection, were rinsed three times with PBS and fixed for $60 \mathrm{~min}$ at $4{ }^{\circ} \mathrm{C}$ with $4 \%$ PFA plus $0.1 \%$ glutaraldehyde and $0.2 \%$ picric acid in 0.1 M sodium cacodylate buffer, $\mathrm{pH}$ 7.2. After rinsing, the cells were dehydrated throughout an ascending methanol series and then embedded in Lowicryl resin (Hespanhol et al. 2005). The ultrastructural immunocytochemistry was performed in the unstained ultrathin sections that were blocked with $50 \mathrm{mM} \mathrm{NH}_{4} \mathrm{Cl}$ and incubated for $20 \mathrm{~min}$ at RT in a blocking buffer containing 4\% BSA plus $1 \%$ Tween 20. The samples were then incubated for $60 \mathrm{~min}$ at RT with affinity purified rabbit anti-TcPRAC IgG. After washing, the samples were incubated for $30 \mathrm{~min}$ at $37^{\circ} \mathrm{C}$ with the goat anti-rabbit IgG coupled to 15-nm colloidal gold particles (Sigma Chemical Co, St. Louis, MO, USA). Uranyl-stained grids were analyzed using an EM10C Zeiss transmission electron microscopy. The primary antibodies were omitted or the samples were incubated with rabbit non-immunized sera. We did not observed non-specific binding of the primary or the secondary antibody, as indicated in the figures when appropriate. 


\section{RESULTS}

Localization of TCPRAC during the T. cruzi life cycle - Previous studies indicated that $T$. cruzi differentially expresses both intracellular and secreted forms of the TcPRAC, possibly in a life stage specific manner (Reina-San-Martin et al. 2000, Chamond et al. 2005). In order to analyze in more detail the sub-cellular localization of TcPRAC during the life cycle, and particularly in the intracellular form of the parasite in epithelial cells of the African green monkey, we used affinity purified rabbit polyclonal IgG raised against TcPRAC that recognizes both isoforms of the enzyme in immunofluorescence experiments. The analysis by confocal laser scanning microscopy associated with differential contrast interference confirmed that similarly to epimastigote (Fig. 1A-C) and metacyclic trypomastigote (Fig. 1D) insect stages, TcPRAC localizes in the cytoplasm of intracellular amastigote forms of the parasites (Fig. 2). The intensity of anti-TcPRAC labeling varied according to the time post infection reaching the highest signal of TcPRAC expression after $48 \mathrm{~h}$, while the number of intracellular parasites increased by amastigote multiplication (Fig. 2D). The analysis by fluorescent microscopy of released parasites (trypomastigotes and amastigotes) after $96 \mathrm{~h}$ of infection showed a positive, but weaker diffuse labeling (not shown). The negative control where the primary antibody was omitted did not show any labeling (Fig. 2A). Non infected Vero cells are not labeled by anti-TCPRAC antibodies or by the goat anti-rabbit IgG-TRITC, as shown in Fig. 2B. In ultrastructural analysis using rabbit anti-PRAC IgG developed with coloidal gold-goat-anti-rabbit antibodies, the immunolabeling (Fig. 3 A-D) confirmed the cytoplasmatic enzyme lo-
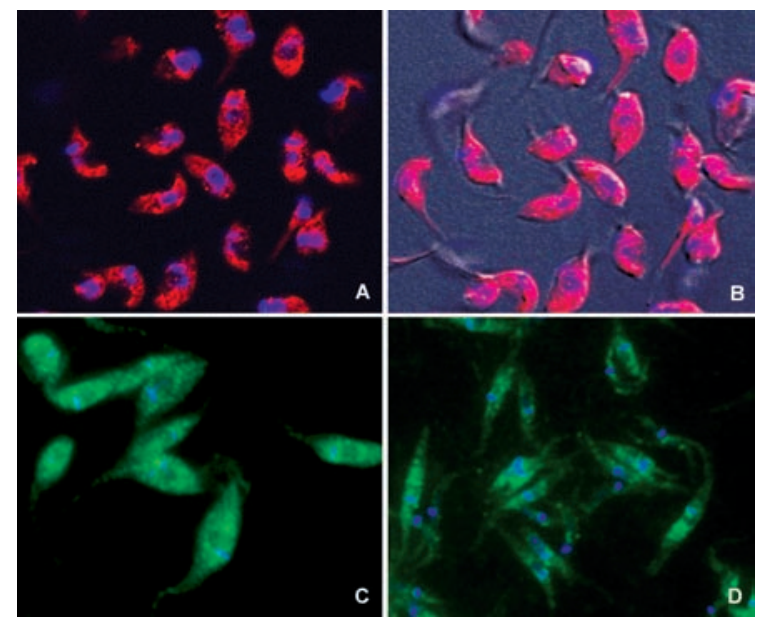

Fig. 1: analysis of TCPRAC expression in the epimastigote (A-C) and metacyclic trypomastigote (D) forms of Trypanosoma cruzi. Confocal laser scanning microscopy and differential contrast interference merge analysis. A-B: epimastigote forms were incubated with tetramethyl rhodamine isothiocyanate-labeled goat anti-rabbit IgG followed by 4,6-diamidino-2-phenylindole (DAPI) staining (blue, revealing parasite nuclei and the stronger labeled kinetoplast). Lower panel: epimastigote (C) and metacyclic trypomastigote forms (D) were incubated with fluorescein isothiocyanate-IgG-labeled goat anti-rabbit IgG followed by DAPI staining. calization: colloidal gold particles were found dispersed in the cytoplasm and within vesicles present nearby the flagellar pocket in epimastigotes or in recently released trypomastigotes (not shown). When non-immune serum was used, no labeling was found (Fig. 3E).

Anti-TcPRAC specific antibodies reduce parasite infection of Vero cells - Since the overexpression of the TcPRAC enzyme in metacyclic forms potentiates their infectivity (Chamond et al. 2005), we next investigated the possible participation of TcPRAC during the invasion of tissue culture derived trypomastigotes into the host cells. These assays were performed by adding anti-proline racemase antibodies during the interaction between trypomastigotes and host cells. When increasing doses of the specific antibody $(42,84$ and $168 \mu \mathrm{g} /$ $\mathrm{mL}$ ) were added to the culture medium, an important dose-dependent decrease in both the percentage of infected Vero cells as well as in the mean number of parasites per infected host cell could be noticed (Fig. 4 A-B) as measured $24 \mathrm{~h}$ after infection. A light microscopy

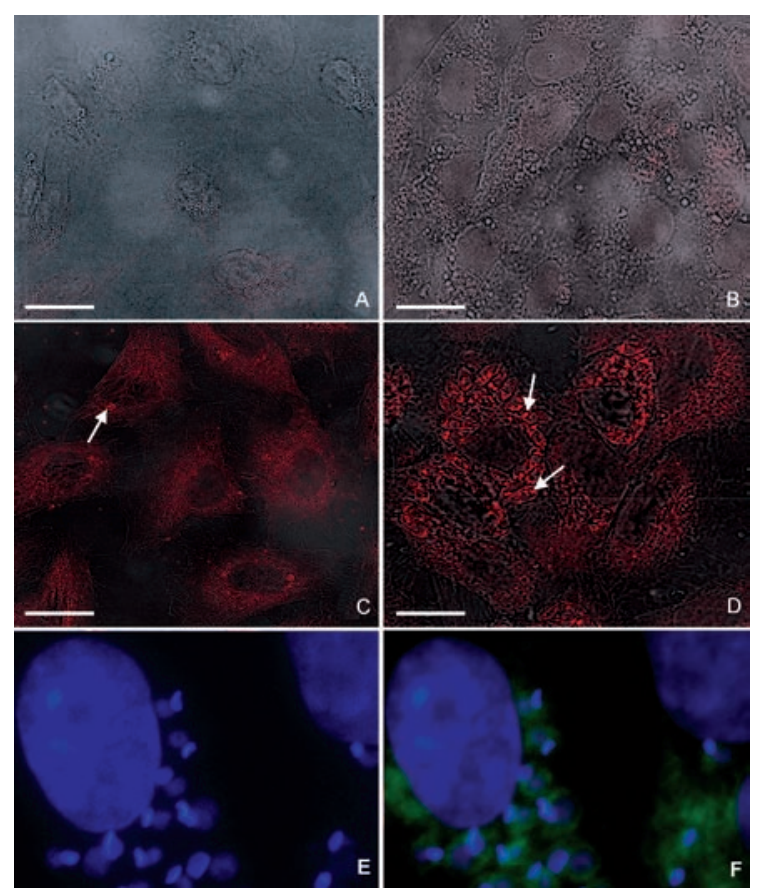

Fig. 2: analysis of TcPRAC expression during the intracellular cycle of Trypanosoma cruzi. Confocal laser scanning microscopy and differential contrast interference merge analysis (A-E). A: uninfected Vero cell cultures were incubated with rhodamin labeled goat anti-rabbit IgG; B: uninfected Vero cell cultures were incubated with rabbit antiTcPRAC IgG antibodies followed by rhodamin labeled goat anti-rabbit IgG; C-F: infected Vero cell cultures were incubated with rabbit anti TcPRAC antibodies that recognize both TcPRACA and TcPRACB isoforms of the protein and further developed by rhodamin-labeled (C-D) or fluorescein isothiocyanate-IgG-labeled (E, F) goat anti-rabbit IgG and 4,6-diamidino-2-phenylindole staining (E, F) - T. cruziinfected Vero cell cultures after $24 \mathrm{~h}(\mathrm{C}), 48 \mathrm{~h}(\mathrm{D})$ or $96 \mathrm{~h}(\mathrm{E}, \mathrm{F})$ of parasite interaction. Often strong labeling can be observed for some amastigotes (arrows). Note that Vero cells do not possess receptor for Fc of immunoglobulins and that the whole affinity purified IgG was used in the experiments. Bars (panels A-D) $=10 \mu \mathrm{m}$. 

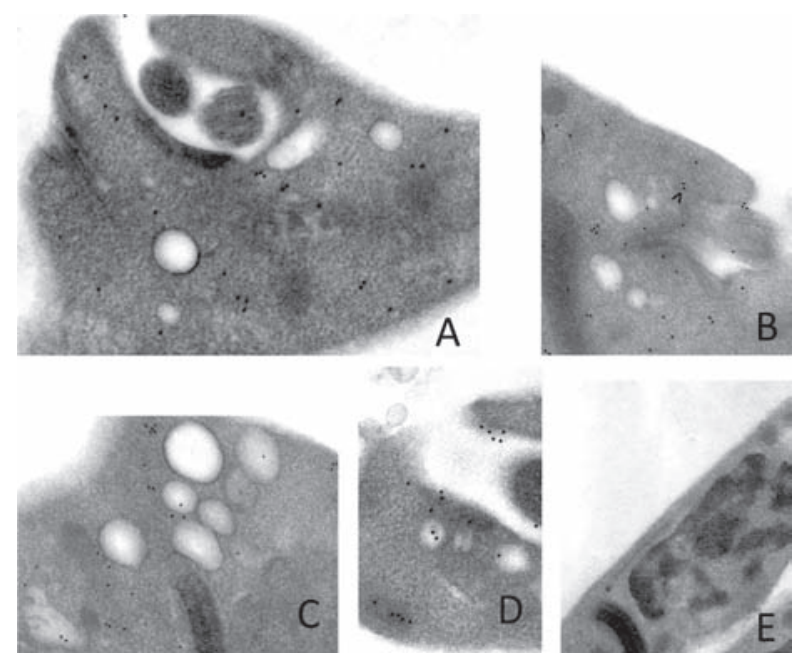

Fig. 3: ultrastructural imunolabeling of epimastigote parasite forms confirming the cytoplasmatic enzyme localization. Colloidal gold particles were found dispersed at the cytoplasm and within vesicles present nearby the flagellar pocket. Samples were incubated with affinity purified rabbit anti-TcPRAC IgG, followed by revelation with goat anti-rabbit IgG coupled to 15-nm colloidal gold particles (A-D). Cells incubated with non-immune rabbit serum did not show any labeling (E).

analysis shows the untreated cells (Fig. 4C) and with the addition of anti-TcPRAC at $168 \mu \mathrm{g} / \mathrm{mL}$ (Fig. 4D). The latter condition lowered by nearly $60 \%$ the number of infected cells ( $\mathrm{p} \leq 0.000029)$ and by $64 \%$ the number of parasites per host cell, while addition of rabbit non immune serum did not induce any alteration on the parasites invasion profile (Fig. 4E).

A specific inhibitor of TcPRAC reduces host cell invasion by T. cruzi - Previous crystallographic data have shown that the incubation of TcPRAC with its competitive inhibitor promotes considerable alteration of the enzyme structure, thus interfering with its binding to B-cell expressed ligands (Buschiazzo at al. 2006). It is worth noting that TcPRAC enzymatic activity only accepts free $\mathrm{L}$ and $\mathrm{D}$-proline, thus excluding the interconversion by this enzyme of proline residues bound to peptide chains expressed by the host cells. To investigate the possible effect of TcPRAC on parasite invasion, we added increasing concentrations of PYC in supernatants of parasite invasive assays using adherent Vero cell cultures. Our results showed that when increasing PYC concentrations $(10,100,1.000 \mu \mathrm{M})$ were added concomitantly with the interaction of trypomastigotes and Vero host cells at a 10:1 cell ratio in vitro, a clear dose-dependent effect was noticed with less infected host cells and lower mean numbers of parasites per infected cell, as measured $24 \mathrm{~h}$ after the onset of interaction. Thus, a significant decrease in the IE (Fig. 5) $(\mathrm{p} \leq 0.0001)$ was observed when the culture cells were treated with higher doses, reaching $86 \%$ of inhibition (Fig. 5C). Control samples treated with comparable amounts of DMSO did not show alterations in the analyses (Fig. 5C). A light microscopy analysis illustrates the dose dependent effect of PYC treatment, in $10 \mu \mathrm{M}$ (Fig. 5E), $100 \mu \mathrm{M}$ (Fig. 5E, F) and $1.000 \mu \mathrm{M}$ (Fig.
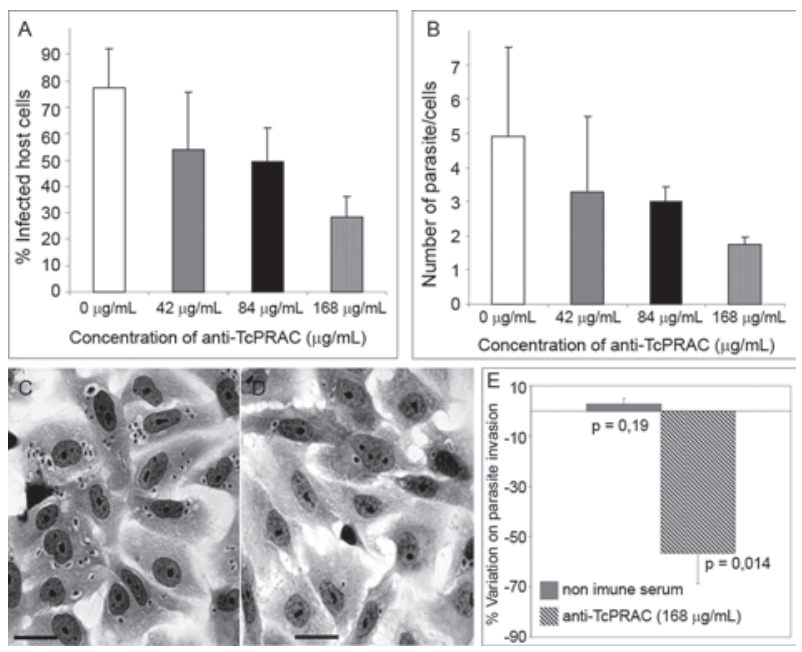

Fig. 4: anti-TcPRAC antibodies hamper the T. cruzi-host cell interaction. A and B show the frequencies observed of host cell infection in cultures where the parasite invasion was realized in the presence of affinity purified rabbit anti-TcPRAC antibodies (A) and the number of parasites per infected cell (B) after $24 \mathrm{~h}$ of interaction performed in the present of increasing doses $(42 \mu \mathrm{g} / \mathrm{mL}, 84 \mu \mathrm{g} / \mathrm{mL}$ and $168 \mu \mathrm{g} / \mathrm{mL})$ of purified rabbit anti-TcPRAC polyclonal antibodies. The data show a dose-dependent inhibition on both the mean number of infected host cells and on the number of parasites per host cell, reaching 59\% (A) and 64\% (B) of decrease, respectively. Light microscopy of untreated (C) and purified rabbit anti-TcPRAC polyclonal antibodies $(168 \mu \mathrm{g} / \mathrm{mL})$ treated cell cultures (D) after $24 \mathrm{~h}$ of infection show a decrease in parasite invasion due to treatment with anti-TcPRAC. E: the negative control performed by the addition of non-immune serum, which did not induce alteration on the parasite invasion. Bars (panels C and D) $=10 \mu \mathrm{m}$.

5E, G), demonstrating a significant decrease in both the mean number of parasites per host cell and in the number of infected host cells (arrows) when they are compared with the untreated sample (Fig. 5D).

Inhibition effect of PYC in pre-treated parasites or host cells before their interaction - In order to clarify whether the interference of proline racemase inhibitor with parasite invasion could be related to an effect of PYC on the host cell or on the TcPRAC enzyme, we performed invasion assays after pre-treatment of the parasites or of the Vero cells for $2 \mathrm{~h}$ before their interaction, using different doses of PYC $(10,100$ and $1.000 \mu \mathrm{M})$. Our data revealed that the pre-treatment of the parasites with $1.000 \mu \mathrm{M}$ of PYC did not lead to changes in their morphology and motility, but resulted in an up to 54\% reduction in the percentage of parasitized cells (Fig. 6A) and about 30\% less parasites per cell (Fig. 6B) when cultures were counted at day 4 after infection. This resulted in an up to $96 \%$ reduction in the number of released trypomastigotes in the supernatant at this time-point (Fig. 6C). When the Vero cells were pre-treated with the inhibitor, no alteration of parasite invasion was observed (Fig. 7A, B), which also demonstrated the lack of toxicity of PYC on the cells under these experimental conditions. Negative controls adding DMSO to the medium in equivalent concentrations after PYC dilution did not alter any of the infectivity parameters (Figs 6, 7). 

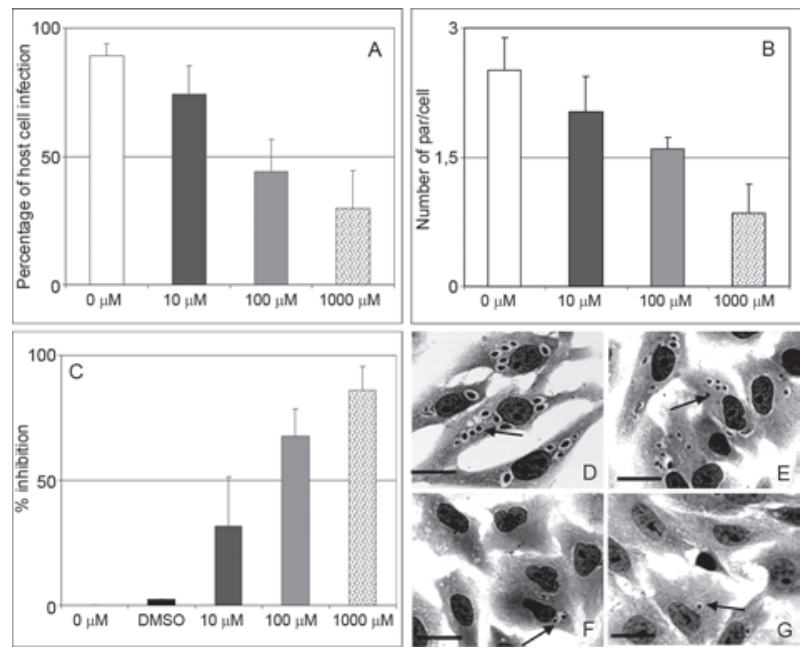

Fig. 5: effect of the pyrrole-2-carboxylic acid (PYC) inhibitor on Trypanosoma cruzi-host cell interaction. A-G: Vero cell cultures were previously infected for $24 \mathrm{~h}$ with trypomastigote culture forms of T. cruzi in the presence or not of different concentrations of PYC $(0-1.000 \mu \mathrm{M})$. A dose-dependent effect of PYC was noticed in the percentage of infected host cells (A), in the number of the parasites per infected cells (B) and thus in the endocytic index. The control samples treated only with dimethyl sulfoxide (DMSO) did not show alterations in the analyses (A-C). Light microscopy of infected Vero cell cultures left untreated (D) or submitted to treatments with 10 (E), $100(\mathrm{~F})$ and $1.000 \mu \mathrm{M}$ PYC (G). The data shows a decrease in both host cell infection and in the number of parasites (arrow) per cell already in cultures treated with low concentrations of the inhibitor (i.e. $10 \mu \mathrm{M}$ final concentrations), as compared to untreated samples (D). Bars (panels D-G) $=10 \mu \mathrm{m}$.

Effect of PYC on T. cruzi intracellular differentiation - Our results indicated that TcPRAC localizes in the cytoplasm of intracellular amastigotes. Thus, we also investigated whether inhibition of the intracellular enzyme would influence parasite differentiation, more precisely from amastigote to trypomastigote forms. For this purpose, Vero cells were infected for $24 \mathrm{~h}$ and washed to eliminate extracellular parasites. Different PYC concentrations $(10,100$ and $1.000 \mu \mathrm{M})$ were added to the medium and the infection was followed for $96 \mathrm{~h}$. In untreated cultures, a significant decrease in the number of amastigote parasites was seen with time, as expected, due to their natural differentiation into trypomastigotes (Fig. 8A, B). However, in the cultures treated with PYC, there was a dose-dependent increase in the percentage of amastigotes thus suggesting that proline racemase activity might be involved in the process of differentiation of intracellular parasites to trypomastigotes (Fig. 8A, C).

\section{DISCUSSION}

Several mechanisms have been reported regarding the invasion of host cells by T. cruzi, involving diverse receptor-ligand systems, triggering signaling cascades and leading to actin-dependent and independent internalization processes (Rosestolato et al. 2002, Woolsey et al. 2003, De Souza et al. 2005, Villalta et al. 2008, Yoshida \& Cortez 2008). In the present study, we analyzed the involvement of proline racemase during the invasion and differentiation steps of the parasite and verified the localization of proline racemase during the cell cycle of $T$. cruzi. TcPRAC is a homodimeric enzyme, whose monomers are folded in two symmetric subunits separated by a deep crevice (Buschiazzo et al. 2006). The enzyme is believed to be expressed as two isoforms: an intracellular and a secreted form (Reina-San-Martin et al. 2000). Previous studies demonstrated that TcPRAC is a potent host B cell polyclonal activator, which contributes to the prevention of specific humoral immune responses in early infection and is thus crucial for parasite evasion and fate (Minoprio 2001). Recent studies have also suggested that the enzyme, through the availability of D-proline in the cytoplasm, allows for further addition of D-proline to parasite peptide chains (Coatnoan et al. 2009). Thus, the presence of D-proline in expressed T. cruzi proteins could bring additional advantage to the parasite by contributing to the initial lack of specificity of host B-cell responses and to the improved parasite resistance to host cellular or extracellular proteases.

Both fluorescent and ultrastructural analysis showed the localization of proline racemase in the cytoplasm but also near the flagellar pocket of the parasites. The latter is a specialized region of the plasma membrane involved in endocytosis and exocytosis of kinetoplastid protozoans. Expression and possible secretion of proline racemase by intracellular amastigotes might possibly contribute to proline sequestration by the parasite. We have previously shown that $T c$ PRAC secreted by metacyclic parasites is involved as a mitogen for host $\mathrm{B}$. It is also conceivable that the release of TCPRAC by T. cruzi amastigotes liberated by disrupted host cells may also contribute to the maintenance of B cell polyclonal lymphocyte activation and thus ensure parasite escape, persistence and pathology associated with the chronic form of Chagas disease (Minoprio 2001, Chamond et al. 2003).

In the present study we show that the intensity of the fluorescence in the intracellular amastigotes was stronger after $48 \mathrm{~h}$ of parasite interaction when, in this experimental model, the parasites are in the process of active multiplication. The expression of TcPRAC decreases later on, with the onset of amastigote to trypomastigote differentiation (not shown). Interestingly, we found that the addition of the specific proline racemase inhibitor PYC in T. cruzi-infected cultures resulted in a dose-dependent decrease of the conversion of amastigotes to trypomastigotes (Fig. 8), suggesting that this enzyme, which is up-regulated in actively multiplying parasites, may be related to parasite differentiation triggering steps. This corroborates our previous results demonstrating that the over-expression of TcPRAC results in an increase in epimastigote differentiation into infective metacyclic forms and that a critical impairment of parasite viability could also be noticed in functional knock-down parasites (Chamond et al. 2005).

The addition of specific anti-proline racemase antibodies (Fig. 4) or the PYC inhibitor (Fig. 5) during the initial steps of parasite-host cell interaction led to a significant initial reduction of $T$. cruzi invasion in vitro. Proline racemase does not act on proline incorporated 

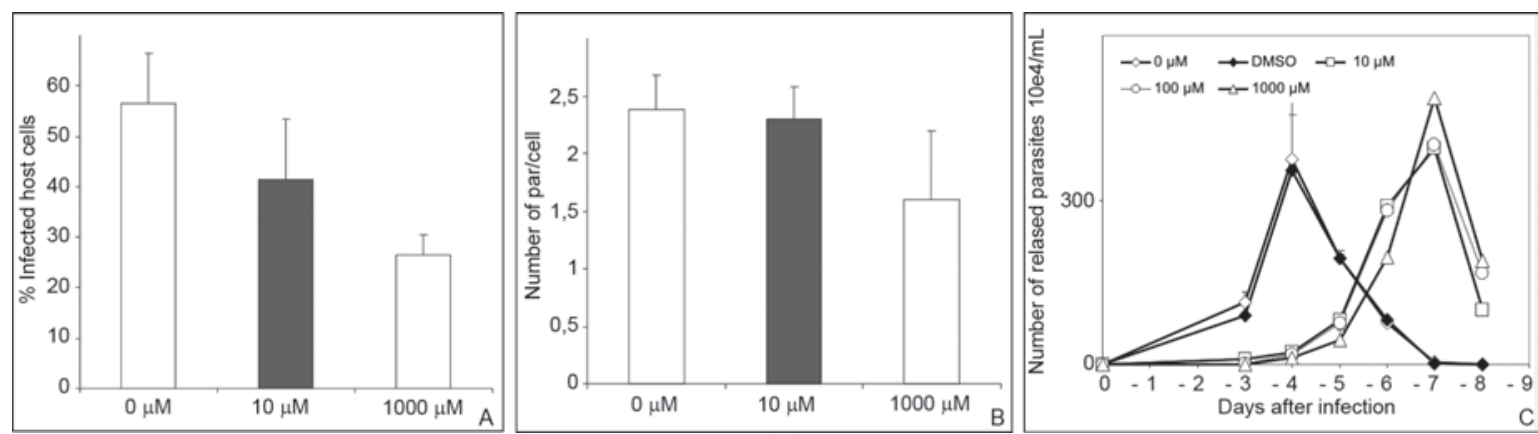

Fig. 6: effects of the pre-treatment of Trypanosoma cruzi with pyrrole-2-carboxylic acid (PYC) on parasite invasion. Cellular infection was scored after $24 \mathrm{~h}$ of Vero cell infection (A-B) and regularly after and up to eight days post infection (C). Trypomastigote forms of the parasite were pretreated for $2 \mathrm{~h}$ with different concentrations of PYC $(10 \mu \mathrm{M}, 100 \mu \mathrm{M}$ or $1.000 \mu \mathrm{M})$ before their interaction with the host cells. The results show a dose-dependent effect on both the percentage of infected host cells (A) and the mean number of parasites per cell (B). After four days, cultures infected with pre-treated parasites present a mean number of released parasites in the supernatants that is significantly reduced (C) when compared to cultures incubated with dimethyl sulfoxide (DMSO) alone that depicted similar levels of released parasites as compared to untreated samples.

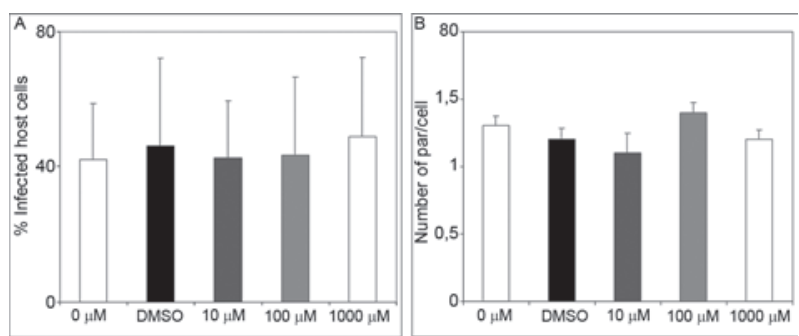

Fig. 7: effects of the pre-treatment of Vero cells with pyrrole-2-carboxylic acid (PYC) on Trypanosoma cruzi invasion. The host cells were pre-treated for $2 \mathrm{~h}$ with different concentrations of PYC $(0 \mu \mathrm{M}, 10 \mu \mathrm{M}$, $100 \mu \mathrm{M}, 1.000 \mu \mathrm{M})$ before their interaction with the trypomastigote parasites (A-B). The results show no significant differences on both the percentage of infected host cells (A) and the mean number of parasites per cell (B) between treated and untreated cultures. Note that mammalian cells do not possess proline racemase genes, corroborating the absence of effect of PYC on a putative host enzyme. The results also show an absence of toxicity of PYC toward Vero cells. The treatment of host cells with dimethyl sulfoxide (DMSO) also did not show any difference in both analyses (A and B), as compared to untreated samples.

into polypeptides, but the inhibition of the enzyme has a direct or indirect influence on the parasite metabolism, differentiation and virulence. The previous incubation of host cells with PYC did not alter the infectious process. However, treatment of trypomastigotes with PYC showed a significant influence of the TcPRAC inhibition in the reduction of parasite invasion. These results indicate that PYC may additionally affect intracellular TcPRAC and consequently interfere with metabolic pathways, differentiation or with the expression of parasite epitopes implicated with invasion. Furthermore, it is worth recalling that $T$. cruzi host cell invasion is dependent on components of the extracellular matrix (Ortega-Barria \& Pereira 1991, Herrera et al. 1994). It is also possible that TcPRAC allows for the modification of cellular matrix components facilitating parasite adhesion. In similar experiments reported by Moro et al. (1995), the involvement of a T. cruzi peptidyl-prolyl

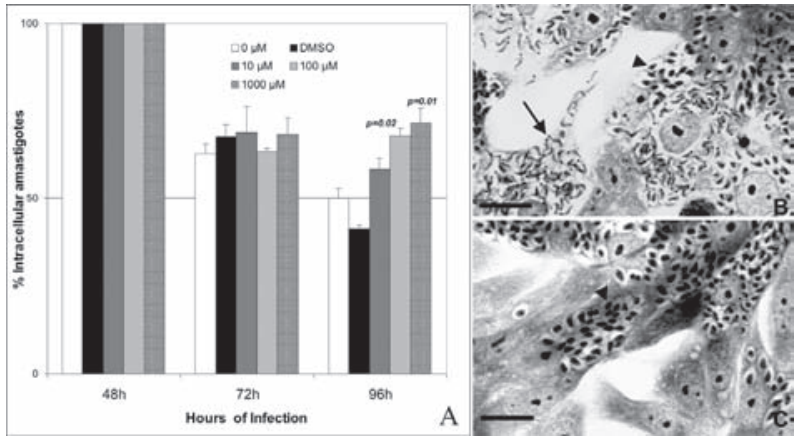

Fig. 8: effect of the pyrrole-2-carboxylic acid (PYC) inhibitor on Trypanosoma cruzi intracellular differentiation. Vero cell cultures were infected for $24 \mathrm{~h}$ with trypomastigote forms, washed to remove free parasites and then maintained in the continuous presence or in the absence of different concentrations of PYC $(10 \mu \mathrm{M}, 100 \mu \mathrm{M}$ and 1.000 $\mu \mathrm{M})(\mathrm{A})$. As expected, after $96 \mathrm{~h}$ of infection a decrease in the percentage of amastigotes, due to their natural conversion to trypomastigote forms, can be noticed in untreated cultures, while a dose-dependent increase of amastigote numbers is observed in the PYC-treated cultures (A). Light microscopy of cells after $96 \mathrm{~h}$ of infection of untreated (B) as compared to the cultures developed in the presence of 1.000 $\mu \mathrm{M}$ PYC (C) shows that untreated cells display higher levels of tripomastigotes (arrows) than inhibitor-treated cells that present higher numbers of amastigote forms. DMSO: dimethyl sulfoxide.

cis-trans isomerase (TcMIP) during the infection of host cells by trypomastigotes was demonstrated. Similar to our assays, these authors demonstrated that antibodies raised against TcMIP significantly inhibited parasite infection possibly impairing the ability of the secreted form of the enzyme to interact with host cell surface components (Moro et al. 1995).

Corroborating the data on the implication of TcPRAC, the specific inhibitor PYC considerably reduced the ability of $T$. cruzi to invade host cells supporting the idea that proline racemase activity is important for the recognition process of the parasite by host cell ligands. These results extend our previous observations, which showed that 
metacyclic trypomastigotes overexpressing TcPRAC are more infective as compared to the wild-type parasites (Chamond et al. 2005). Although previous work of the our group has shown that the TcPRAC-PYC interaction has an influence on the overall structure of the protein precluding its interaction with B-cell expressed ligands (Buschiazzo et al. 2006), the exact mechanism regarding TcPRAC involvement in T. cruzi invasion of host cells still remains unknown. Additionally, our present data indicate that the PYC inhibitor hampers intracellular amastigote to trypomastigote differentiation (Fig. 8), which no doubt would contribute to diminished parasite development and fate. We found further evidences that the enzyme localizes nearby and in the flagellar pocket of the parasite forms, which can give rise to important repercussions for the immune system responses during the parasite infection in both acute and late phases of the disease. As a result, reduced levels of parasite specific responses throughout infection by mitogenic stimulation at the tissue environment may certainly contribute to parasite persistence and consequently to progressive pathology and tissue damage.

Our present data substantiate earlier findings showing that proline racemase plays a role in both infectivity and differentiation of T. cruzi (Chamond et al. 2005) and support its use as a critical lead for the development of chemotherapy against Chagas disease. PYC is a known inhibitor of proline racemases with very poor solubility in water and our recent efforts using medicinal chemistry to synthesize more soluble PYC variants have not yielded better inhibitors of TcPRAc with a higher affinity for the enzyme (manuscript in preparation). However, molecular dynamics studies based on PYC were used to design pharmacophoric models to screen libraries of chemical compounds. The results of those studies will certainly be important to allow further and better appreciation of TcPRAC impact in vivo.

\section{REFERENCES}

Araujo-Jorge TC, Sampaio EP, De Souza W, Meirelles MN 1989. Trypanosoma cruzi: the effect of variations in experimental conditions on the levels of macrophage infection in vitro. Parasitol Res 75: 257-263.

Bilate AM, Teixeira PC, Ribeiro SP, Brito T, Silva AM, Russo M, Kalil J, Cunha-Neto E 2008. Distinct outcomes of Trypanosoma cruzi infection in hamsters are related to myocardial parasitism, cytokine/chemokine gene expression and protein expression profile. J Infect Dis 198: 614-623.

Buschiazzo A, Goytia M, Schaeffer F, Degrave W, Shepard W, Gregoire C, Chamond N, Cosson A, Berneman A, Coatnoan N, Alzari PM, Minoprio P 2006. Crystal structure, catalytic mechanism and mitogenic properties of Trypanosoma cruzi proline racemase. Proc Natl Acad Sci USA 103: 1705-1710.

Chamond N, Goytia M, Coatnoan N, Barale JC, Cosson A, Degrave WM, Minoprio P 2005. Trypanosoma cruzi proline racemases are involved in parasite differentiation and infectivity. Mol Microbiol 58: 46-60.

Chamond N, Gregoire C, Coatnoan N, Rougeot C, Freitas-Junior LH, Da Silveira JF, Degrave WM, Minoprio P 2003. Biochemical characterization of proline racemases from the human protozoan parasite Trypanosoma cruzi and definition of putative protein signatures. J Biol Chem 278: 15484-15494.
Coatnoan N, Berneman A, Chamond N, Minoprio P 2009. Proline racemases: insights into Trypanosoma cruzi peptides containing D-proline. Mem Inst Oswaldo Cruz 104 (Suppl.): 295-300.

Coura JR, Junqueira AC, Fernandes O, Valente AS, Miles MA 2002. Emerging Chagas disease in Amazonian Brazil. Trends Parasitol 18: 171-176.

Covarrubias C, Cortez M, Ferreira D, Yoshida N 2007. Interaction with host factors exacerbates Trypanosoma cruzi cell invasion capacity upon oral infection. Int J Parasitol 37: 1609-1616.

Da Silva CF, Batista MM, Batista DG, De Souza EM, Da Silva PB, De Oliveira GM, Meuser AS, Shareef AR, Boykin DW, Soeiro MN 2008. In vitro and in vivo studies of the trypanocidal activity of a diarylthiophene diamidine against Trypanosoma cruzi. Antimicrob Agents Chemother 52: 3307-3314.

De Souza EM, Araujo-Jorge TC, Bailly C, Lansiaux A, Batista MM, Oliveira GM, Soeiro MN 2003. Host and parasite apoptosis following Trypanosoma cruzi infection in in vitro and in vivo models. Cell Tissue Res 314: 223-235.

De Souza VA, Da Silva R, Pereira AC, Royo VA, Saraiva J, Montanheiro M, De Souza GH, Da Silva Filho AA, Grando MD, Donate PM, Bastos JK, Albuquerque S, Silva ML 2005. Trypanocidal activity of (-)-cubebin derivatives against free amastigote forms of Trypanosoma cruzi. Bioorg Med Chem Lett 15: 303-307.

De Souza W 2008. Electron microscopy of trypanosomes - a historical view. Mem Inst Oswaldo Cruz 103: 313-325.

Dias JC 2006. Chagas disease: successes and challenges. Cad Saude Publica 22: 2020-2021.

Dias JC 2007. Globalization, inequity and Chagas disease. Cad Saude Publica 23 (Suppl. 1): S13-22.

Fernandes MC, Cortez M, Geraldo-Yoneyama KA, Straus AH, Yoshida N, Mortara RA 2007. Novel strategy in Trypanosoma cruzi cell invasion: implication of cholesterol and host cell microdomains. Int J Parasitol 37: 1431-1441.

Herrera EM, Ming M, Ortega-Barria E, Pereira ME 1994. Mediation of Trypanosoma cruzi invasion by heparan sulfate receptors on host cells and penetrin counter-receptors on the trypanosomes. Mol Biochem Parasitol 65: 73-83.

Hespanhol RC, de Nazaré C Soeiro M, Meuser MB, de Nazareth SL, Meirelles M, Côrte-Real S 2005. The expression of mannose receptors in skin fibroblast and their involvement in Leishmania (L.) amazonensis invasion. J Histochem Cytochem 53: 35-44.

Marin-Neto JA, Rassi AJR, Morillo CA, Avezum A, Connolly SJ, Sosa-Estani S, Rosas F, Yusuf S 2008. Rationale and design of a randomized placebo-controlled trial assessing the effects of etiologic treatment in Chagas cardiomyopathy: the benznidazole evaluation for interrupting trypanosomiasis (BENEFIT). Am Heart J 156: 37-43.

Minoprio P 2001. Parasite polyclonal activators: new targets for vaccination approaches? Int J Parasitol 31: 588-591.

Moro A, Ruiz-Cabello F, Fernandez-Cano A, Stock RP, Gonzalez A 1995. Secretion by Trypanosoma cruzi of a peptidyl-prolyl cistrans isomerase involved in cell infection. EMBO J 14: 2483-2490.

Ortega-Barria E, Pereira ME 1991. A novel T. cruzi heparin-binding protein promotes fibroblast adhesion and penetration of engineered bacteria and trypanosomes into mammalian cells. Cell 67: 411-421.

Reina-San-Martin B, Degrave W, Rougeot C, Cosson A, Chamond N, Cordeiro-Da-Silva A, Arala-Chaves M, Coutinho A, Minoprio P 2000. A B-cell mitogen from a pathogenic trypanosome is a eukaryotic proline racemase. Nat Med 6: 890-897. 
Rocha MO, Teixeira MM, Ribeiro AL 2007. An update on the management of Chagas cardiomyopathy. Expert Rev Anti Infect Ther 5: 727-743.

Rosestolato CT, Dutra JM, De Souza W, De Carvalho TM 2002. Participation of host cell actin filaments during interaction of trypomastigote forms of Trypanosoma cruzi with host cells. Cell Struct Funct 27: 91-98.

Schmunis GA 2007. Epidemiology of Chagas disease in non-endemic countries: the role of international migration. Mem Inst Oswaldo Cruz 102 (Suppl. I): 75-85.

Tarleton RL 2007. Immune system recognition of Trypanosoma cruzi. Curr Opin Immunol 19: 430-434.
Villalta F, Madison MN, Kleshchenko YY, Nde PN, Lima MF 2008. Molecular analysis of early host cell infection by Trypanosoma cruzi. Front Biosci 13: 3714-3734.

Woolsey AM, Sunwoo L, Petersen CA, Brachmann SM, Cantley LC, Burleigh BA 2003. Novel PI 3-kinase-dependent mechanisms of trypanosome invasion and vacuole maturation. J Cell Sci 116: 3611-3622.

Yoshida N 2006. Molecular basis of mammalian cell invasion by Trypanosoma cruzi. An Acad Bras Cienc 78: 87-111.

Yoshida N, Cortez M 2008. Trypanosoma cruzi: parasite and host cell signaling during the invasion process. Subcell Biochem 47: 82-91. 